\title{
EFEITO DA CONFIGURAÇÃO DOS INJETORES NA HOMOGEINIZAÇÃO DO CATALISADOR NO REATOR RISER FCC
}

\author{
PELISSARI, D.C ${ }^{1}$, ALVAREZ C, H.C. ${ }^{1}$, MORI $\mathrm{M}^{1}{ }^{1}{ }^{*}$, MARTIGNONI, $\mathrm{W}^{2}$ \\ ${ }^{1}$ Universidade Estadual de Campinas, Departamento de Engenharia Química \\ ${ }^{2}$ PETROBRAS/AB-RE-TR/OT \\ E-mail para contato: danielcicero21@ @otmail.com; mori@ feq.unicamp.br
}

\begin{abstract}
RESUMO - O processo de craqueamento catalítico fluido (FCC) é uma das aplicações de fluidização gás-sólido mais importante na indústria de petróleo. Na operação do riser, a carga é alimentada através de injetores e misturada com catalisador e vapor de fluidização. A performace dos injetores para garantir uma evaporação rápida do gasóleo e uma boa distribuição das gotículas com o catalisador é importante para melhorar a eficiência do processo e assim, promover uma distribuição de reagentes uniforme no riser ajudando a garantir bons resultados na conversão da carga e no rendimento dos produtos. O principal objetivo deste trabalho foi avaliar o efeito do design e da configuração dos injetores sobre a homogeneidade do gás-sólido através da fluidodinâmica computacional (CFD) usando modelo cinético de 12-lumps. As equações foram resolvidas com uma abordagem Euleriana-Euleriana 3D usando o solver CFX versão 14.0 como ferramenta de cálculo. Os resultados das simulações mostraram que a distribuição de gás-sólido depende significativamente da configuração dos injetores de gasóleo. Acredita-se que é a primeira vez que é aplicado CFD para avaliar o design dos bicos no interior do riser na literatura aberta.
\end{abstract}

\section{INTRODUÇÃO}

A unidade de craqueamento catalítico fluidizado (UFCC) converte diversos subprodutos do petróleo de baixo valor comercial em derivados (gasolina, diesel, gases leves, entre outros) de maior valor agregado, sendo considerado um dos processos mais importantes e rentáveis da refinaria de petróleo por, sendo assim, estudada por diversos pesquisadores a fim de compreender melhor os fenômenos que ocorrem no processo. A UFCC pode ser dividida em três partes: riser, separador e regenerador. Sendo que o craqueamento catalítico ocorre principalmente no riser, o riser da FCC é um longo tubo vertical de alta relação altura/diâmetro, no qual o catalisador e o gasóleo entram em por tempo adequado. A região inferior do riser é conhecida como região lift, nesta parte são introduzidos o catalisador e o vapor de fluidização. O gasóleo é injetado no riser por dispersores de carga, que têm como objetivo atomizar a carga em goticulas pequenas e introduzi-las no riser. Além disso os dispersores de carga tem como função promover a distribuição adequada da carga no interior do riser, para minimizar regiões de elevada concentração de gasóleo ou catalisador melhorando o contato entre as partículas de catalisador e o gasóleo. 


\section{9 a 22 de outubro de 2014 \\ Florianópolis/SC}

A zona de injeção é considerada a região mais complexa do reator, devido a intensa turbulênciae e o regime não homogênio, resultando em elevados gradientes de temperatura e concentração. Segundo Theologos et. al. (1997), Theologos e Markatos (1993), Mauleon e Coorcelle (1985) e Behjat et al. (2010) a geometria dos injetores influência na hidrodinâmica do reator e no craqueamento catalítico, influênciando assim o rendimento.

Theologos et.al. (1997) simulou um riser FCC utilizando um modelo unidimensional com modelo cinético de 10-lumps para descrever as reações de craqueamento catalítco, estudando a influência do número de injetores sobre a peformace do reator. O resultado da simulação mostrou que o rendimento das reações desejadas é melhorado com o aumento do número de injetores, pois proporciona uma distribuição mais homegênea do catalisador.

Lopes et al. (2011) utilizou um modelo tridimensional em escoamento gás-sólido usando um modelo cinético de 4 lumps. Os resultados mostraram também que não uniformidade da distribuição do catalisador afeta o desempenho do reator. Além disso eles salientam que a utilização de geometrias mais próximas com as reais gerou uma distribuição das fases mais próximas com as encontradas na literatura.

Jiang Li (2013) utilizou um modelo de 14-lump para simular o riser FCC. O resultado da simulação mostrou que a velocidade de injeção da matéria-prima e o ângulo de injeção da mesma tem influência no processo, enquanto que a posição dos injetores não apresentou influência significativa.

Visto que a injeção de gasóleo através dos bicos injetores afeta o desempenho do riser, e consequentemente o rendimento ecônomico das UFCC, o presente trabalho estudo a influência do injetores sobre a fluidodinâmica do riser de FCC, estudando bicos internos e diferentes designs, com a finalidade de melhorar a distribuição do catalisador no riser.

\section{MODELAGEM MATEMÁTICA}

Foi empregado um modelo 3-D para descrever os fenômenos de transporte do escoamento, com uma abordagem Euleriana-Euleriana usando as equações de conservação de massa, momento e energia, o modelo $k-\epsilon$ para modelar a turbulência da fase gasosa, Ranz-Marshall para o cálculo do Nusselt e Gidaspow (1994) para definir as interações gás-partícula, estas últimas correlações apresentadas na Tabela 1. Além disso, foi utilizado o modelo cinético de 12-lumps desenvolvido por $\mathrm{Wu}$ (2009) para representar as reações. Nas equações apresentadas $\varepsilon, \rho$ e $u$ são a fração volumétrica, densidade e velocidade para cada fase respectivamente; $\mu$ é a viscosidade; $\lambda, h_{g s}$, a condutividade térmica e o coeficiente de transferência de calor entre as fases; $G$ é o módulo de elasticidade; $\beta$ a transferência de momento na interfase; $C_{D}$ é o coeficiente de arraste; $d_{s}$ é o diâmetro da partícula sólida; $R e_{s}$ é o número de Reynolds; $N u$ é onúmero de Nusselt; e (Pr) é o número de Prandtl. Os subscritos $g$ e $s$ representão a fase gás e a fase sólida, respectivamente. 
Tabela 1- Equações que governam a simulação

Força de arraste (Gidaspow 1994)

$\beta= \begin{cases}150 \frac{\varepsilon_{s}^{2} \mu_{g}}{\varepsilon_{g} d_{s}^{2}}+\frac{7}{4} \frac{\left|u_{s}-u_{g}\right| \varepsilon_{s} \rho_{g}}{d_{s}} & \varepsilon_{s}>0,2 \\ \frac{3}{4} C_{D} \frac{\left|u_{s}-u_{g}\right| \varepsilon_{s} \varepsilon_{g} \rho_{g} \varepsilon_{g}^{-2,65}}{d_{s}} & \varepsilon_{s}<0,2\end{cases}$

$C_{D}= \begin{cases}0,44 & R e_{s}>1000 \\ \frac{24}{R e_{s}}\left[1+0,15\left(R e_{s}\right)^{0,687}\right] & R e_{s}<1000\end{cases}$

Pressão do sólido (Gidaspow 1994)

$G=G_{0} \exp \left[c\left(\varepsilon_{s}-\varepsilon_{s, \max }\right)\right]$

Coeficiente de tranferência de calor entre as fases

$h_{g s}=\frac{\lambda_{g} \cdot N u}{d_{p}}$

Correlação de Ranz-Marshall

$N u=2,0+0,6 \cdot R e^{0,5} \cdot \operatorname{Pr}^{0,33}$

\section{SIMULAÇÃO}

A geometria do riser e os design dos bicos, bem como suas dimensões, são apresentados na Figura 1, enquanto que as condições operacionais podem ser vista na Tabela 2. Avaliou-se quatro casos neste trabalho, sendo o caso 1 sem bicos internos e os demais casos com bicos internos e diferentes designs. As malhas computacionais empregadas nas simulações variaram de aproximadamente 675 mil à 985 mil elementos, adequadas para simular risers industriais de FCC como descrito no trabalho de Alvarez-Castro et al (2011).Utilizou-se condição de não deslizamento na parede para ambas as fases. Empregou-se o esquema de interpolação de alta resolução, com critério de convergência RMS de $10^{-4}$. O tempo de simulação foi de $15 \mathrm{~s}$, onde os primeiros $10 \mathrm{~s}$ foram para a estabilização do sistema e os 5 s finais para a obtenção das médias aritiméticas. 
Tabela 2-Condições operacionais

Temperatura de reação $(\mathrm{K})$

Queda de pressão $(\mathrm{kPa})$

Tempo de reação (s)

Fluxo de gasóleo (t/h)

Temperatura de entrada do gasóleo (K)

Temperatura de entrada do catalisador $(\mathrm{K})$

Relação catalisador/gasóleo
793,15

124,46

543,15

913,15

8,1
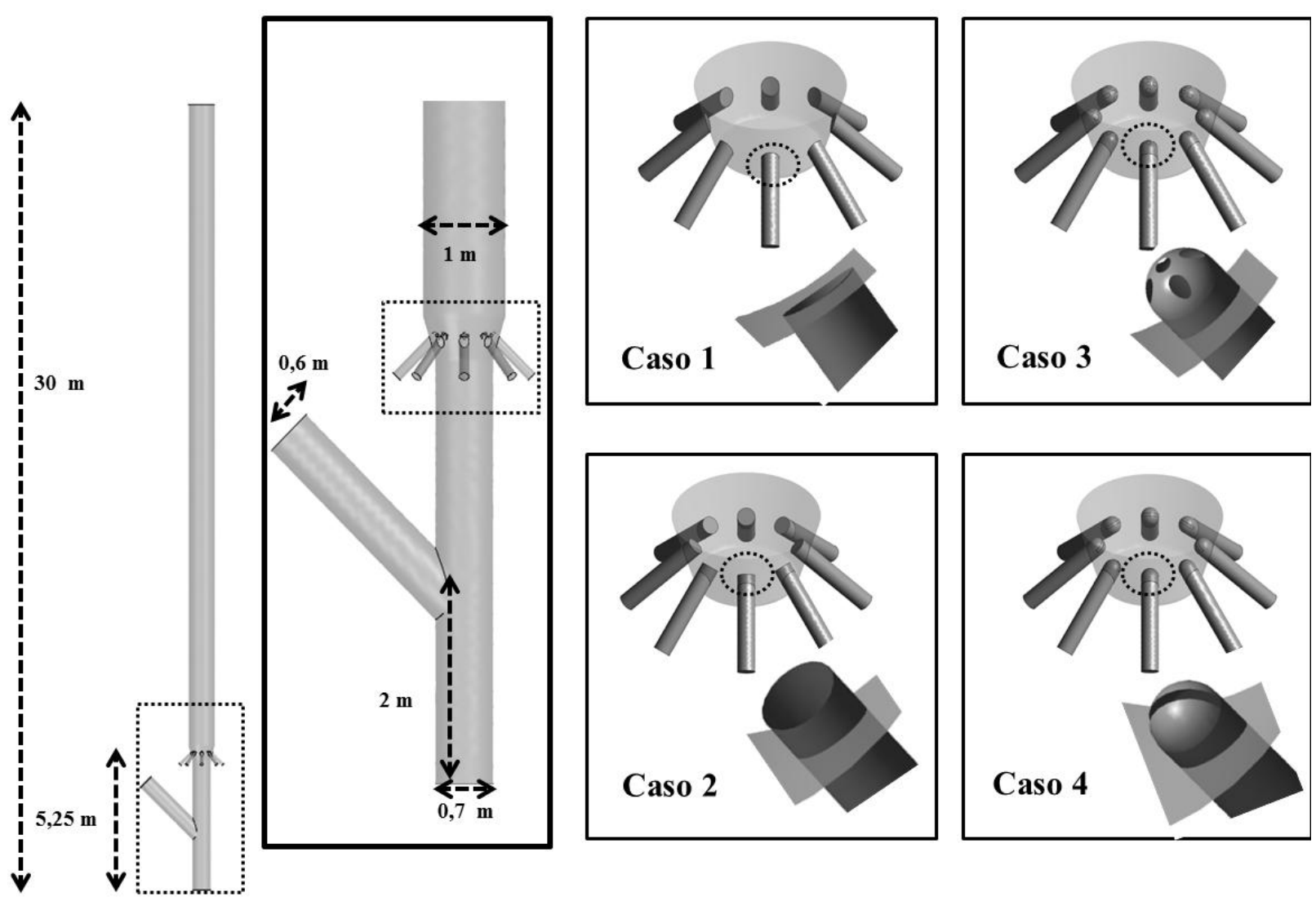

Figura 1-Geometria do riser e design dos bicos 


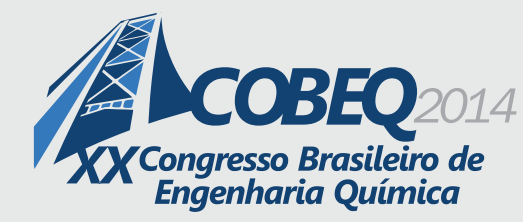

19 a 22 de outubro de 2014
Florianópolis/SC

\section{RESULTADOS}

A Figura 2 mostra a fração de sólido em seções tranversais em diferentes alturas ao longo do riser. Pode-se ver em todos os casos que para a altura de 4,5 m (antes dos injetores), o catalisador apresenta um grande acúmulo na parede oposta a entrada do catalisador, no entanto, nota-se que na altura de 5,3 m, logo após os injetores, este conseguem redistribuir totalmente as partículas de catalisador com o gasóleo, mostrando com isso a importância dos bicos injetores. Também observa-se que para o caso 1, o qual não possuí bicos interno, que este difere dos demais casos, sendo que os casos com bicos internos mostram o acúmulo de catalisador na parede (core-annules). Nota-se ainda que dentro dos casos com injetores interno, o design dos mesmos afetam a significativamente a fluidodinâmica do processo.

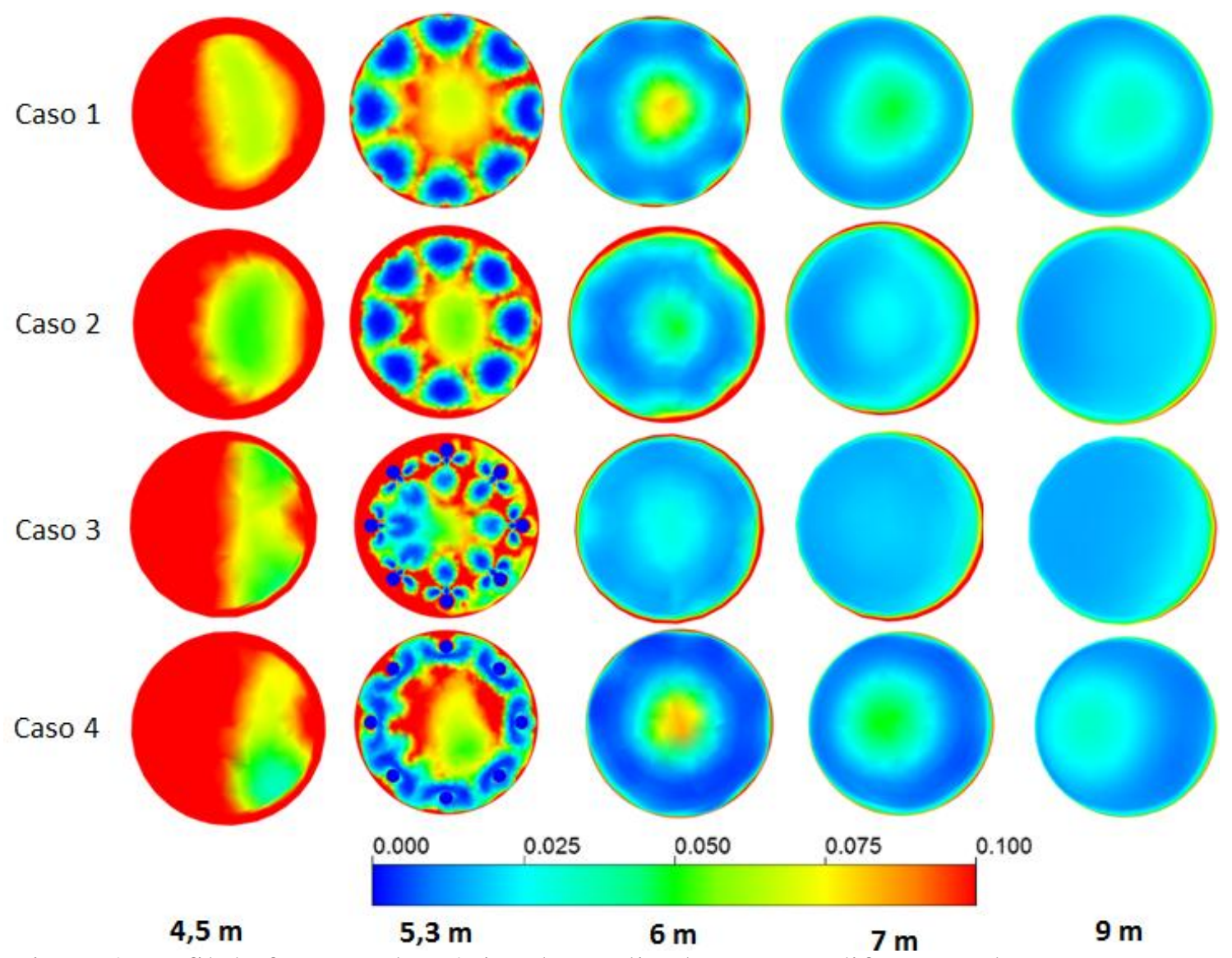

Figura 2-Perfil da fração volumétrica de catalisador para as diferentes alturas

Nas figuras 3 e 4 é apresentado os histograma das médias da fração volumétrica do catalisador para os planos nas alturas 6 me $9 \mathrm{~m}$, respectivamente, onde a linha vermelha representa a média da distribuição do catalisador na área transversal na mesma altura para cada caso e as barras azuis indicam a quantidade de catalisador na coordenada radial. Pode-se observar que na altura de $6 \mathrm{~m}$ o caso que apresenta melhor homegeneização é o caso 2, enquanto o caso 4 apresenta a pior 
homogeneização. No entanto, pode-se ver que na altura 9 m o caso 3 apresenta melhor distribuição de catalisador, enquanto o caso 1 apresenta a menor homogeneização, como pode ser obersavado na figura 5 .

Nota-se ainda que apesar dos injetores internos apresentarem um maior acúmulo de catalisador na parede logo após a injeção do gasóleo, observa-se na Figura 4 que na altura de 9 m, todos os casos possuem uma fração volumétrica de catalisador semelhante próxima a parede, contudo os casos com bicos internos apresentaram um melhor homogeneização.
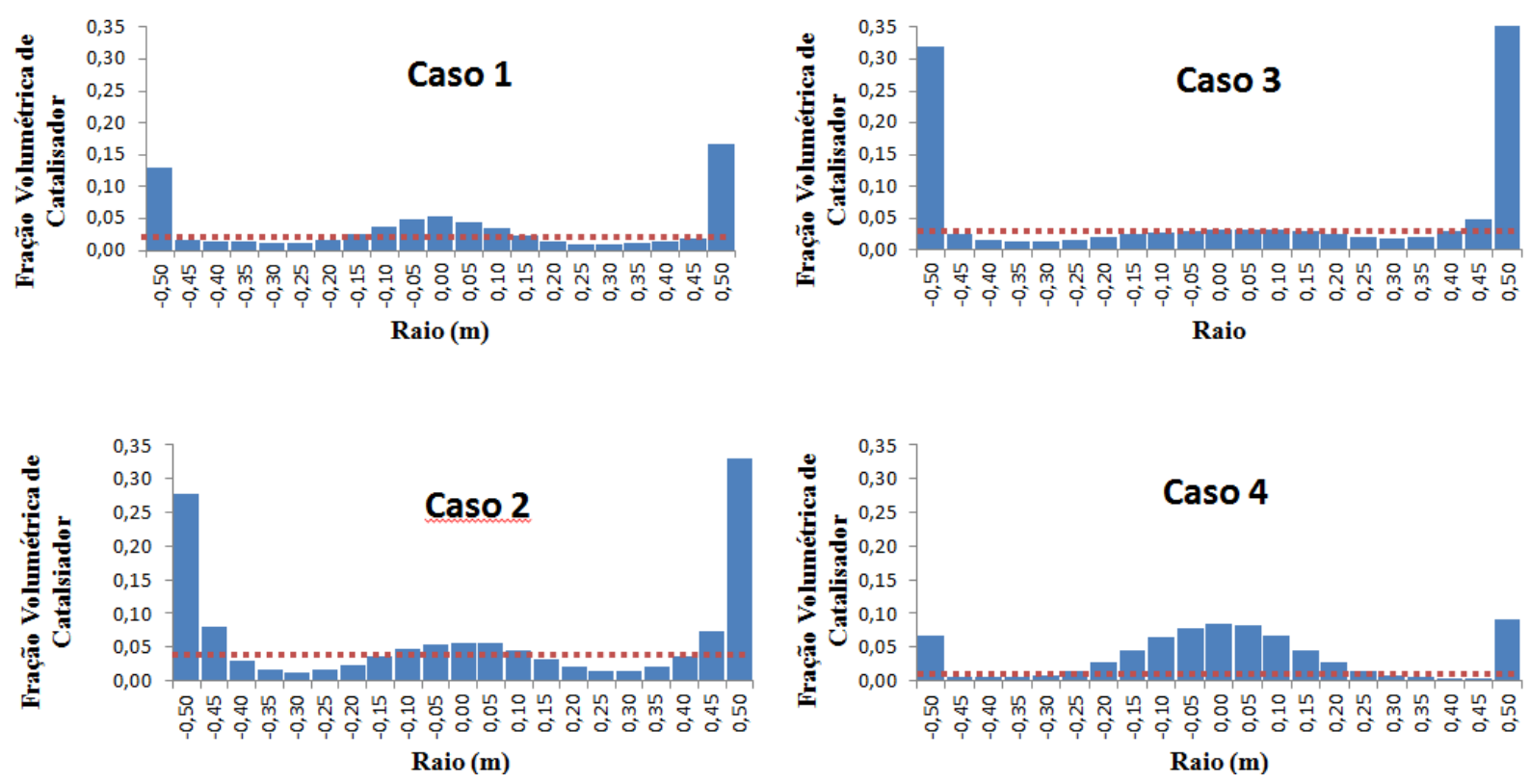

Figura 3-Distrubuição radial da fração volumétrica de catalisador (altura $6 \mathrm{~m}$ ) 

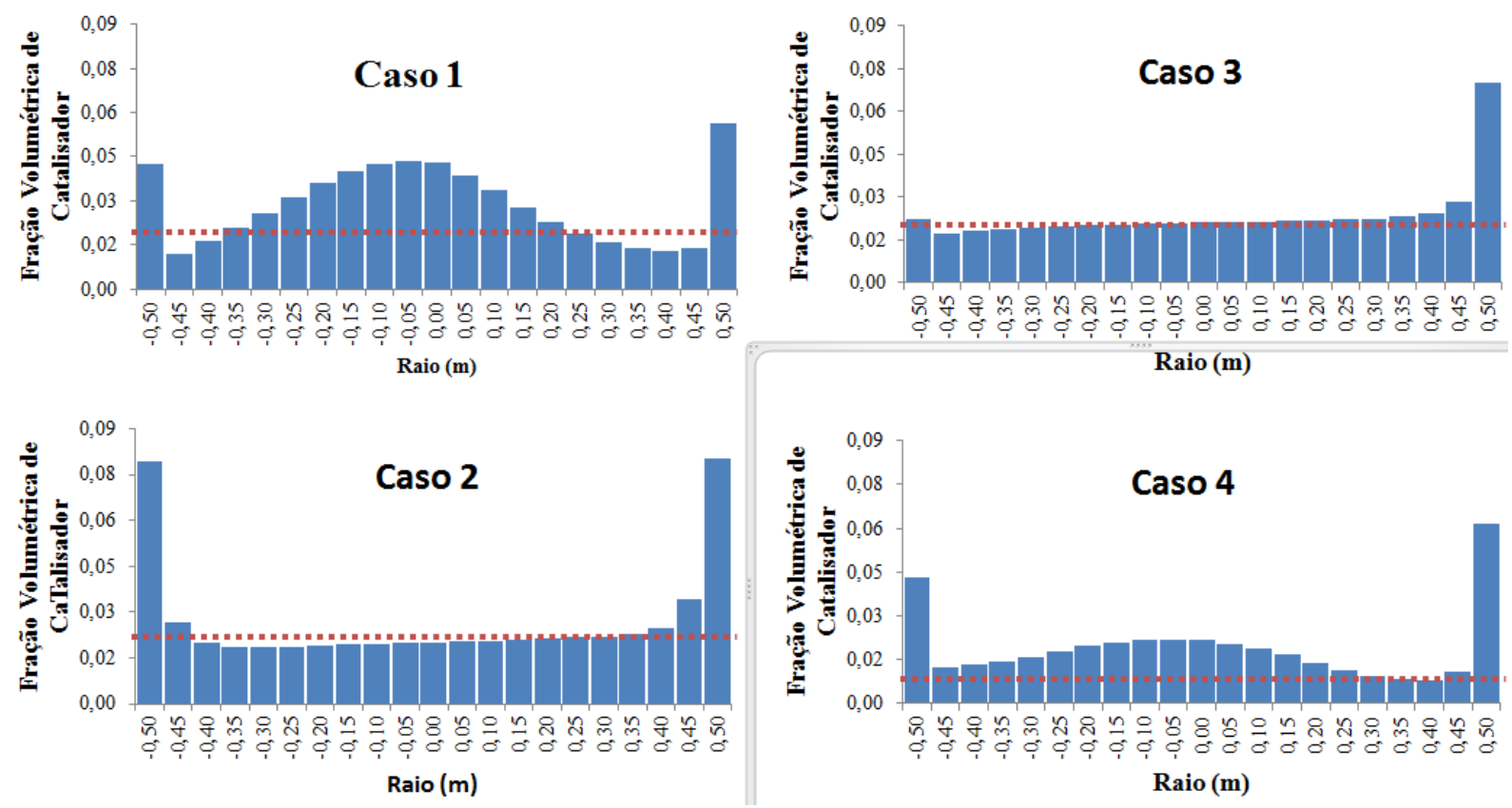

Figura 4-Distrubuição radial da fração volumétrica de catalisador (altura 9 m)

\section{CONCLUSÕES}

Diante dos resultados das simulações, nota-se que para o melhoramento do processo de FCC faz-se necessário a utilziação de bicos injetores, já que estes afetam de forma significativa a interação gás-sólido

$\mathrm{Na}$ avaliação dos bicos injetores no riser, pode-se ver como estes tem uma influência significativa sobre a hidrodinâmica. Sendo que o caso 1, simulado com bicos sem parte interna dentro do riser tem um perfil diferente quando comparado com os casos 2, 3 e 4 com bicos internos no riser. Além disto pode-se observar que para os casos estudados o caso 3 é que apresenta uma melhor distribuição de gasóleo catalisador no riser.

\section{TRABALHOS FUTUROS}

Dentro do estudo também encontrou-se que dependendo o design do bico interno ele consegue uma hidrodinâmica mais adequada na homogeneização do catalisador no riser o que nos permite continuar avaliando diversos variáveis dentro dos bicos injetores como são ângulos, formatos e posições.

\section{AGRADECIMENTOS}

Os autores agradem a CAPES e Petrobras pelo apoio financeiro nesta pesquisa 


\section{REFERÊNCIAS}

ALVAREZ C, H.C., MATOS E.M, MORI M, MARTIGNONI, W. 3D CFD mesh configurations and turbulence models studies and their influence on the industrial risers of fluid catalytic cracking. AIChE annual meeting in Pittsburgh. USA. 2012.

BEHJAT, Y.; SHAHHOSSEINI, S.; MARVAST, M.A. Modeling gas oil spray coalescence and vaporization in gas solid riser reactor. Int. Comm. in Heat and Mass Transfer, v.37, p.935943, 2010.

GIDASPOW, D. Multiphase flow and fluidization: Continuum and kinetic theory descriptions. Academic Press, San Diego, 1994.

LI, J.; FAN, Y.P.; LU, C.X.; LUO, Z.H. Numerical simulation of influence of feed injection on hydrodynamic behavior and catalytic cracking reactions in a FCC riser under reactive conditions. Ind. Eng. Chem. Re, v. 52, p. 11084-11098, 2013.

LOPES, G.C.; ROSA, L.M.; MORI, M.; NUNHEZ, J.P.; MARTIGNONI, W.P. The importance of using three-phase 3-D model in the simulation of industrial FCC risers. Chem. Eng. Trans., v.24, p.1417-1422, 2011.

MAULEON, J.L.; COURELLE, J.C. FCC heat balance critical for heavy fuels. Oil Gas J, v.83, p.64-70, 1985.

THEOLOGOS, K.N; MARKATOS, N.C. Advanced Modeling os fluid catalytic cracking risertype reactors. AIChE J., v.39, p. 1007-1017, 1993

THEOLOGOS, K.N; NIKOU, I.D; LYGEROS, A.I; MARKATOS, N.C. Simulation and design of fluid catalytic cracking riser-type reactors. Comp. Chem. Eng., v.20, p. 757-762, 1997.

WU, F.Y. Study on lumped kinetic model for FDFCC. Unpublished doctoral dissertation. East China University of Science and Technology, China, 2009. 